\title{
Research Paper \\ The Relationship between Work-Related Musculoskeletal Disorders and Types of Used Treatment Methods Among Iranian Physiotherapists
}

\author{
Mahdi Rahmati-Yami ${ }^{1}\left(\mathbb{D}\right.$, Elham Azarmi ${ }^{1}$, Leila Rahnama ${ }^{1}$, Samaneh Hosseinzadeh ${ }^{2},{ }^{*}$ Noureddin Karimi ${ }^{1}$
}

1. Department of Physiotherapy, University of Social Welfare and Rehabilitation Sciences, Tehran, Iran 2. Department of Biostatistics, University of Social Welfare and Rehabilitation Sciences, Tehran, Iran.

\begin{tabular}{|l|l|l|l|l}
\hline $\begin{array}{c}\text { Use your device to scan } \\
\text { and read the article online }\end{array}$ & $\begin{array}{l}\text { Citation Rahmati-Yami M, Azarmi E, Rahnama L, Hosseinzadeh S, Karimi N. [The Relationship Between Work-Related } \\
\text { Musculoskeletal Disorders and Types of Used Treatment Methods Among Iranian Physiotherapists (Persian)]. Archives of Reha- } \\
\text { bilitation. 2020; 21(1):22-39. https://doi.org/10.32598/RJ.21.1.2538.2 }\end{array}$ \\
dol https://doi.org/10.32598/RJ.21.1.2538.2
\end{tabular}

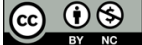

Received: 18 Nov 2018 Accepted: 06 Apr 2019 Available Online: 01 Apr 2020

Keywords:

Physiotherapist, Workplace, Cumulative trauma disorder, Work-related musculoskeletal disorders, Risk factors

\begin{abstract}
Objective After respiratory disorders, musculoskeletal disorders are the second prevalent cause of short-term sickness absence (less than 2 weeks). The nature of the work in physiotherapy requires physical activities, which include repetitive skills, manual techniques, putting direct pressure on a particular joint, unskilled posture during special maneuvers, and having long postural positions. Several studies have reported that physiotherapists frequently experience musculoskeletal disorders. This study aims to determine the relationship between Work-Related Musculoskeletal Disorders (WRMDs) and the common therapeutic methods used by Iranian physiotherapists.

Materials \& Methods This is a descriptive-analytical epidemiological study with a cross-sectional design. The study population consists of physiotherapists working in Iran. Data collection tools included a demographic form, as well as general Nordic questionnaire designed by Kuorinka et al. to detect the WRMD symptoms in 9 body areas in the past year. The questionnaires were distributed among participants in cooperation with the Iranian Physiotherapy Association during the $27^{\text {th }}$ Iranian Physiotherapy Congress. Data analysis was performed in SPSS using the Chi-square and Fisher exact tests. Results Of 1200 questionnaires distributed among physiotherapists, 685 questionnaires were returned after completion. Of these, 16 were excluded due to missing some inclusion criteria, 4 due to having a work experience less than one year, and 11 due to having other diseases, such as neurological and rheumatic diseases, malignancies, and joint replacement. The mean $\pm S D$ age of the participants was $37.9 \pm 9.91$ years ranged from 22 to 69 years; $53.9 \%$ of them were female and 46.1\% male. After obtaining the prevalence of WRMDs, its significant association with the type of used treatment method was examined by the Fischer exact test. The results showed a significant correlation of WRMDs with treatment methods in 7 out of 9 areas. In particular, WRMDs were associated with manual massage $(P=0.001)$, myofascial release $(P=0.011)$, mobilization $(P=0.007)$, muscle energy technique $(P=0.007)$, and dry needling $(P=0.032)$ in the neck area; with mobilization $(P=0.005)$ and taping $(P=0.014)$ in the shoulder area. Also, WRMDS are associated with respiratory physiotherapy $(P=0.007)$ in elbows. WRMDS are correlated with manual massage $(P=0.027)$, myofascial release $(P=0.001)$, mobilization $(P=0.046)$, muscle energy technique $(P=0.004)$, and taping $(P=0.016)$ in wrists/hands. In the upper back, WRMDs are associated with manual massage $(P=0.007)$, mobilization $(P=0.014)$, and taping $(P=0.004)$. In the lower back, WRMDs are seen with
\end{abstract}

* Corresponding Author:

Noureddin Karimi, PhD.

Address: Department of Physiotherapy, University of Social Welfare and Rehabilitation Sciences, Tehran, Iran.

Tel: +98 (912) 2573865

E-Mail: karimi@uswr.ac.ir 
myofascial release $(P=0.036)$, mobilization $(P=0.015)$, taping $(P=0.035)$, and muscle energy technique $(P=0.044)$. Finally, WRDMs are observed with water therapy $(P=0.037)$ in knees.

Conclusion There is a high prevalence of WRMDs among physiotherapists in Iran. Its rate has a significant relationship with the type of treatment methods. Some physiotherapy methods can increase the prevalence of WRMDs.

\section{Extended Abstract}

\section{Introduction}

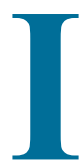

$\mathrm{n}$ the workplace, human beings are affected by various harmful factors such as ergonomic, physical, chemical, etc. All of these factors cause fatigue, early weakness, and, in economic terms, a waste of time and money [1]. The term "musculoskeletal disorders" refers to a large group of inflammatory and destructive diseases that affect the muscles, ligaments, tendons, joints, intervertebral disks, nerves, and blood vessels [2, 3]. Work-related musculoskeletal disorders (WRMDs) are associated with risk factors in the workplace and are known by various names such as cumulative trauma disorders and repetitive traction injuries [4]. In today's world, the issue of prevention and control of WRMDs is extremely important because a large part of the compensation paid to the injured workforce belonged to these disorders. Studies have shown that more than half of the absences in the workplace are caused by musculoskeletal disorders [5].

Work-related injuries can lead to problems such as job loss, job restrictions, fatigue, burnout, or eventually change of job [6]. WRMDs are among the most important causes of occupational injury and disability in industrialized and developing countries $[7,8]$. Improper body posture and lack of knowledge about the correct principles of work are among the most important causes of WRMDs. These disorders are the main occupational problems among health care providers [9-12].

Physiotherapy is one of the occupations in the field of rehabilitation that is prone to WRMDs for various reasons, such as direct contact with patients, different activities, and physical conditions during work [13]. The rate of these injuries among physiotherapists in the UK has been reported 68\% [14]; in Australia, 55\% [15]; in Turkey, 85\% [16]; in Nigeria, 91.3\% [17]; and in Greece, 89\% [18]. In Iran, Sharhaninezhad et al. (2014) conducted a study in Ahvaz City and their findings indicated a high prevalence of musculoskeletal problems among physical therapists [19]. Nazari et al. (2016) conducted a study in the cities of Hamedan, Nahavand, and Malayer. They reported a prevalence rate of $58.3 \%$ among physical therapists [20]. Several studies have suggested that WRMDs have been frequently experienced by physiotherapists [21]. Recent evidence suggests that the prevalence of work-related injuries in therapists is increasing every year [6]. Overall, it is estimated that the direct and indirect costs of musculoskeletal disorders may account for about $1 \%$ of the gross domestic product of industrialized countries [22].

Information about work-related injuries among physiotherapists is limited $[6,15,18]$. Considering that there is no study on work-related musculoskeletal problems among physiotherapists in Iran, we aimed to investigate the relationship between WRMDs with various types of treatment used by physiotherapists in Iran.

\section{Materials and Methods}

This is a descriptive-analytical epidemiological study with a cross-sectional design. The study population consists of all physiotherapists working in Iran in 2016. According to the Iranian Physiotherapy Association, the study population was 3600 . Of these, 1200 physiotherapists participating in the $27^{\text {th }}$ Iranian Physiotherapy Congress were selected. The inclusion criteria were having academic education in physiotherapy with at least a bachelor's degree, at least 1 year of work experience, and without musculoskeletal disorders before engaging in physiotherapy. The exclusion criteria were unwillingness to continue participation and having a disease in other systems of the body, including neurological and rheumatic diseases, malignancies, and joint replacement. After distributing 1200 questionnaires, only 686 were returned. Of these, 16 were excluded due to missing some inclusion criteria; 4 due to having work experience less than 1 year; and 12 due to having other diseases. Finally, 670 physiotherapists participated in the study.

Data collection tools were a demographic form as well as the common therapeutic techniques used by participants (the method used for more than $60 \%$ of their patients), and general Nordic questionnaire for measuring the prevalence of WRMDs. It is a self-report tool that assesses WRMDs in 9 areas of the body (neck, shoulders, upper back, lower back, elbows, wrists/hands, hips/ thighs, knees, and ankles/feet) over 1 year. This questionnaire is one of the most widely-used questionnaires in the field of musculoskeletal disorders, designed in 1987 by Kuorinka et al. [23]. It lacks an overall score and de- 
termines the frequency of injury. For the Persian version of this questionnaire, the internal consistency was found 0.8 and repeatability as a Kappa $>0.7$ at $\mathrm{P}<0.001$ [24].

Before data collection, informed consent was obtained from the participants. They were assured of the confidentiality of their information and were free to leave the study at any time. After collecting data, they were analyzed using the Chi-square and Fisher exact tests in SPSS V. 23. The significance level was set at 0.05 .

\section{Results}

The mean \pm SD age of the participants was $37.09 \pm 9.91$ years ranged 22-69 years (53.9\% females and $46.1 \%$ males). Also, $91.8 \%$ of them were right-handed; $70.3 \%$ had a colleague or assistant in their work environment, and $69.6 \%$ had more than 5 years of experience. Table 1 presents the demographic information of the participants. WRMDs were significantly higher in women compared to men in the neck, shoulders, wrists/hands, upper back, and knees $(\mathrm{P}<0.001)$. There was a significant relationship between age and WRMDs in the elbow $(\mathrm{P}=0.021)$ and upper back $(\mathrm{P}=0.02)$ of the participants.

Table 1. Demographic information of the participants $(n=670)$
Those with ages between 30 and 40 years had the highest rate of WRMDs in the upper back area.

There was no significant association between the prevalence of WRMDs and work experience, having an assistant or colleague, being a right-handed or left-handed, and smoking. The common therapeutic techniques used by the participants were exercise therapy $(89.6 \%)$, electrotherapy $(88.8 \%)$, patient training $(82.2 \%)$, mobilization $(59.4 \%)$, stretching $(58.3 \%)$, manual massage $(46.7 \%)$, dry needling $(40 \%)$, myofascial release $(34.3 \%)$, muscle energy technique (34\%), taping $(33.4 \%)$, massage by a device $(27.2 \%)$, respiratory physiotherapy (26.1\%), manipulation (18.8\%), water therapy (3.8\%), and other methods (12.8\%) (Table 2, Figure 1).

To measure the relationship between these methods provided by physiotherapists and WRMDs, we used the Chi-square and Fisher exact tests. The results showed a significant correlation of WRMDs with treatment methods in 7 out of 9 areas. In particular, WRMDs were associated with manual massage $(\mathrm{P}=0.001)$, myofascial release $(\mathrm{P}=0.011)$, mobilization $(\mathrm{P}=0.007)$, muscle energy technique $(\mathrm{P}=0.007)$, and dry needling $(\mathrm{P}=0.032)$ in the neck area; with mobilization $(\mathrm{P}=0.005)$ and taping $(\mathrm{P}=0.014)$ in

\begin{tabular}{|c|c|c|}
\hline Characteristic & Groups & No. (\%) \\
\hline \multirow[b]{2}{*}{ Gender } & Female & $361(53.9)$ \\
\hline & Male & 309 (46.1) \\
\hline \multirow{2}{*}{ Work experience } & $<5$ years & $204(30.4)$ \\
\hline & $>5$ years & $466(69.6)$ \\
\hline \multirow[b]{2}{*}{ Dominant hand } & Right & $615(91.8)$ \\
\hline & Left & $55(8.2)$ \\
\hline \multirow[b]{2}{*}{ Having an assistant or colleague } & Yes & $471(70.3)$ \\
\hline & No & 199 (29.7) \\
\hline \multirow{2}{*}{ Smoking } & Yes & $80(11.9)$ \\
\hline & No & $590(88.1)$ \\
\hline \multirow{4}{*}{ Age (y) } & $20-30$ & $215(32.1)$ \\
\hline & $31-40$ & $236(35.2)$ \\
\hline & $41-50$ & $146(21.8)$ \\
\hline & $>50$ & 73 (10.9) \\
\hline
\end{tabular}


Table 2. Common therapeutic techniques used by the participants

\begin{tabular}{|c|c|}
\hline Common Therapeutic Technique & No. (\%) \\
\hline Electrotherapy & $595(88.8$ \\
\hline Exercise therapy & $600(89.6$ \\
\hline Patient training & $551(82.2$ \\
\hline Stretching & $391(58.4)$ \\
\hline Massage by a device & $1829(27.2)$ \\
\hline Manual massage & $313(46.7)$ \\
\hline Respiratory physiotherapy & $175(26.1)$ \\
\hline Myofascial Release & $230(34.3)$ \\
\hline Mobilization & $398(59.4)$ \\
\hline Manipulation & $126(18.8)$ \\
\hline Muscle energy technique & $228(34.0)$ \\
\hline Taping & $224(33.4)$ \\
\hline Dry needling & $268(40.0)$ \\
\hline Water therapy & $26(3.9)$ \\
\hline Others & $86(12.8)$ \\
\hline
\end{tabular}

Table 3. Correlation between common therapeutic techniques provided by subjects and WRMDs in different areas of the body

\begin{tabular}{|c|c|c|c|c|c|}
\hline \multirow{2}{*}{$\begin{array}{l}\text { Areas } \\
\text { Neck }\end{array}$} & \multicolumn{5}{|c|}{ Common Therapeutic Techniques } \\
\hline & $\begin{array}{l}\text { Manual massage } \\
\qquad P=0.001\end{array}$ & $\begin{array}{l}\text { Myofascial release } \\
\qquad P=0.011\end{array}$ & $\begin{array}{l}\text { Mobilization } \\
\quad \mathrm{P}=0.007\end{array}$ & $\begin{array}{l}\text { Muscle energy tech- } \\
\text { nique } \mathrm{P}=0.007\end{array}$ & $\begin{array}{c}\text { Dry needling } \\
P=0.032\end{array}$ \\
\hline Shoulder & Mobilization $\mathrm{P}=0.005$ & taping $\mathrm{P}=0.014$ & & & \\
\hline Elbows & $\begin{array}{l}\text { Respiratory physiotherapy } \\
\qquad P=0.007\end{array}$ & & & & \\
\hline Wrists/Hands & Manual massage $P=0.027$ & $\begin{array}{l}\text { Myofascial release } \\
\qquad P=0.001\end{array}$ & $\begin{array}{l}\text { Mobilization } \\
\quad \mathrm{P}=0.046\end{array}$ & $\begin{array}{l}\text { Muscle energy tech- } \\
\text { nique } \mathrm{P}=0.004\end{array}$ & Taping $\mathrm{P}=0.016$ \\
\hline Upper back & Manual massage $P=0.007$ & $\begin{array}{l}\text { Mobilization } \\
\mathrm{P}=0.014\end{array}$ & Taping $\mathrm{P}=0.004$ & & \\
\hline Lower back & $\begin{array}{l}\text { Myofascial } \\
\qquad=0.036\end{array}$ & $\begin{array}{l}\text { Mobilization } \\
\mathrm{P}=0.015\end{array}$ & Taping $\mathrm{P}=0.035$ & $\begin{array}{l}\text { Muscle energy tech- } \\
\text { nique } \mathrm{P}=0.044\end{array}$ & \\
\hline Hips/Thighs & - & & & & \\
\hline Knees & Water therapy $\mathrm{P}=0.037$ & & & & \\
\hline Ankles/Feet & - & & & & \\
\hline
\end{tabular}




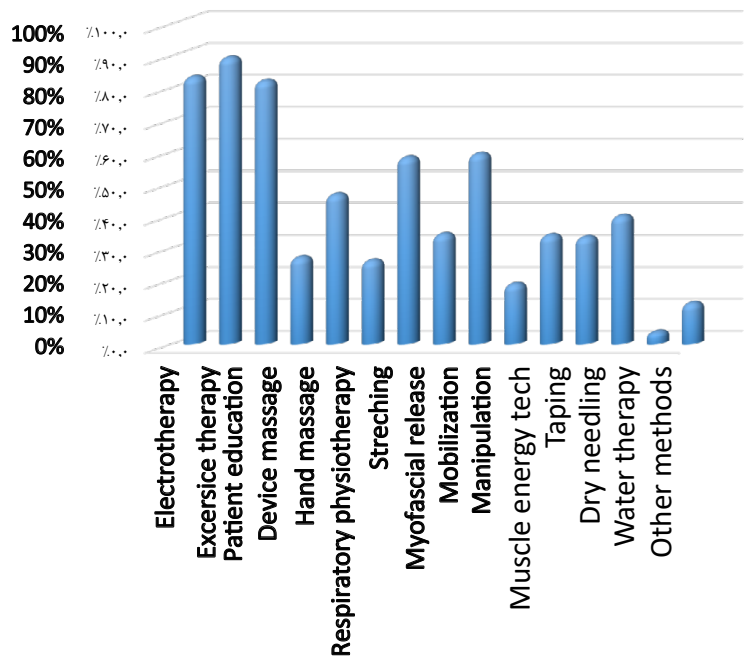

Figure 1. Common therapeutic techniques used by the participants in the past year

the shoulder area. Also, WRMDS are associated with respiratory physiotherapy $(\mathrm{P}=0.007)$ in elbows.

WRMDS are correlated with manual massage $(\mathrm{P}=0.027)$, myofascial release $(\mathrm{P}=0.001)$, mobilization $(\mathrm{P}=0.046)$, muscle energy technique $(\mathrm{P}=0.004)$, and taping $(\mathrm{P}=0.016)$ in wrists/hands. In the upper back, WRMDs are associated with manual massage $(\mathrm{P}=0.007)$, mobilization $(\mathrm{P}=0.014)$, and taping $(\mathrm{P}=0.004)$. In the lower back, WRMDs are seen with myofascial release $(\mathrm{P}=0.036)$, mobilization $(\mathrm{P}=0.015)$, taping $(\mathrm{P}=0.035)$, and muscle energy technique $(\mathrm{P}=0.044)$. Finally, WRDMs are observed with water therapy $(\mathrm{P}=0.037)$ in knees. (Table 3).

\section{Discussion}

The main purpose of this study was to investigate the relationship between work-related musculoskeletal problems and the common therapeutic methods used by the Iranian physiotherapists. There was a significant relationship between gender and incidence of WRMDs, which is consistent with the study of Adegoke et al. They identified female gender as a factor in the development of musculoskeletal disorders [17]. However, it was against the results of Salik and Özcan, Sharhaninezhad et al., and Nazari et al. $[16,19,20]$. Adegoke et al. and Glover et al. reported that in the first 5 years of employment, musculoskeletal injuries are more common $[14,17]$. In our study, there was no relationship between work experience and the higher musculoskeletal disorders which is in agreement with the results of Darragh et al. [6] and Sharhaninezhad et al. [19] studies. In the study of Borke et al. [25], performing manual therapy such as mobilization and working on soft tissue, lifting and transferring patients were among the tasks associated with developing WRMDs. The present study also reported these findings. Joint mobilization (43\%) and manual massage $(41 \%)$ are two factors that can cause musculoskeletal disorders in the wrists and fingers [27]. In our study, mobilization (37\%) and manual massage (38\%) were also responsible factors for musculoskeletal disorders in the wrist area. Manual techniques performed by physiotherapists put stress on the hands and fingers [28]. In Wajon et al. study [29], a significant relationship was found between the use of force and thumb pain during manual therapy [29]. Physiotherapists who use manual techniques are about 3.5 times more likely to hurt their wrists/hands than other therapists [25]. In our study, a significant statistical relationship was found between WRMDs in the wrist area and performing a manual massage, myofascial release, mobilization, muscle energy technique, and taping. This finding agrees with other studies that have linked the use of manual therapy techniques such as massage to wrists and hands symptoms [20, $25,30]$. Alrowayeh et al. [31], however, found no significant association between job factors and increased workrelated musculoskeletal injuries.

Kuehnel et al. [32] in a study compared the prevalence of work-related injuries among college students during manual therapy class in five different continents and found different rates. They indicated that these different rates may be due to cultural differences. For example, the definitions of pain, health, and injury vary in different cultural communities [32]. The point to consider in this study is that the prevalence of work-related musculoskeletal problems among Iranian physiotherapists had a significant relationship with some techniques that they used for treatment such as dry needling and taping. This result indicates that physiotherapists may use incorrect postures to perform these techniques, which can put high pressure on the joints. Amini 
et al. introduced improper handling as the most common cause of injuries in therapists [33]. Rahimi Moghadam et al. stated that with an increase in ergonomics knowledge, the incidence of musculoskeletal disorders in individuals decreases [9].

One of the strengths of this study was its implementation at the national level with a high number of samples (686 physiotherapists). It is also the first study in Iran to examine the relationship between therapeutic methods used by physiotherapists and WRMDs. The limitations of this study were as follows: not including all physiotherapists in the country, using a simple sampling method, and lacking the cooperation of some participants. It is suggested that other studies be conducted in this field using a cluster sampling method at the national level.

\section{Conclusion}

There is a significant relationship between WRMDs and gender, as these disorders are more common in women. This may be due to differences in muscle structure and volume, sex hormones, and biomechanical differences between men and women. There is also a significant relationship between the incidence of these disorders and treatment methods used by physiotherapists. Appropriate decisions need to be made to improve and teach physiotherapists how to manipulate the patients. Teaching physiotherapists to perform the methods correctly, have rest and pause in performing methods that may take a long time, and use existing equipment to prevent occupational injuries can play an important role in reducing their injuries. Increased awareness in the field of ergonomics reduces WRMDs. Training programs can also have a significant impact on increasing the knowledge of correct physical postures while working.

\section{Ethical Considerations}

\section{Compliance with ethical guidelines}

Ethical considerations were observed in the study and ethical approval was obtained from the Research Ethics Committee of the University of Welfare and Rehabilitation Sciences (Code: IR.USWR.REC.1395.66).

Funding

The present paper was extracted from the MA. thesis of Mahdi Rahmati-Yami approved by department of Physiotherapy, University of Social Welfare and Rehabilitation Sciences.

\section{Authors' contributions}

Conceptualization: Noureddin Karimi, Leyla Rahnema, and Mehdi Rahmati Yami; Methodology: Noureddin Karimi, Samaneh Hosseinzadeh, Mehdi Rahmati Yami, and Elham Azarmi; Validation and supervision: Noureddin Karimi, Leyla Rahnema,and Samaneh Hosseinzadeh; data analysis: Leyla Rahnema,and Samaneh Hosseinzadeh, and Mehdi Rahmati Yami; investigation: Leyla Rahnema,and Samaneh Hosseinzadeh, Noureddin Karimi, Mehdi Rahmati Yami, and Elham Azarmi; resources: Mehdi Rahmati Yami, Elham Azarmi, Noreddin Karimi, and Leyla Rahnema; initial draft preparation, editing \& review, visulaization: Mehdi Rahmati Yami and Elham Azarmi; project administration: Noureddin Karimi.

\section{Conflicts of interest}

The authors declared no conflict of interest.

Acknowledgements

The authors would like to thank physiotherapists Dr Bandpey, Dr Abdollahi, Mr Moazzenzadeh, Mr Najafi Sani, Mr Shahrakinasab, Mr Alasti, Dr Ravanbakhsh, Ms Monjazi, Ms Khodadadi, Mr Nourollahzadeh, Ms Naghdi, Ms Abdolalizadeh, Ms Mirshahi, and all participants for their valuable cooperation. 
This Page Intentionally Left Blank 


\title{
بررسى ارتباط مشكلات اسكلتى ـ عضلاتى ناشى از كار با انواع روشهاي درمات دانى در ميان فيزيوتراييستهاى شاغل ايران در سال هوبال
}

\author{
مهدى رحمتى يامى' 1. الهام آزرمى'، ليلا رهنما'، سمانه حسينزاده'، "تورالدين كريمى'
}

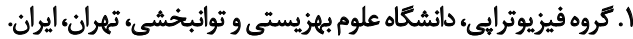

r. أكروه آمار زيستى، دانشكاه علوم بهزيستى و توانبخشى، تهران، ايران.

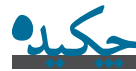

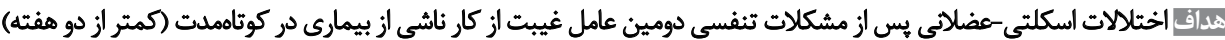

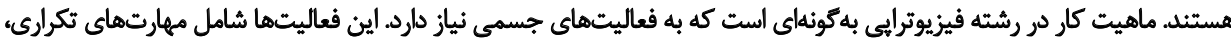

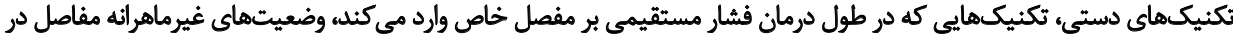

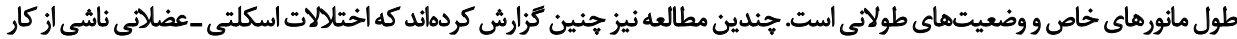

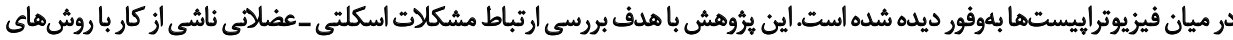

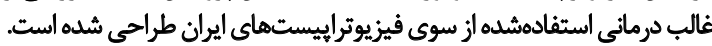

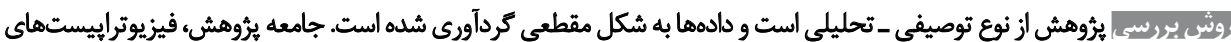

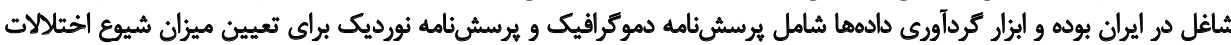

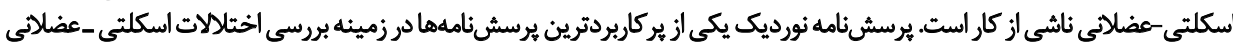

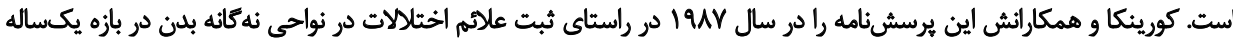

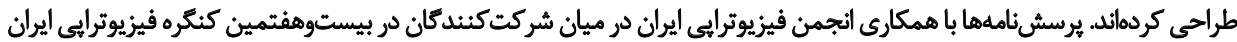

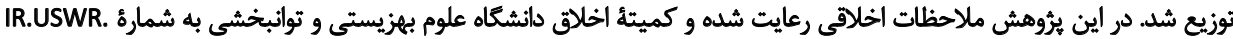

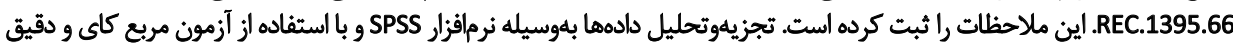

$$
\text { فيشر انجام شده است. }
$$

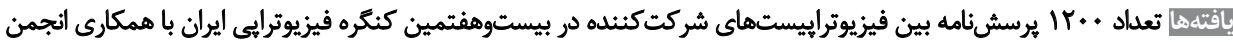

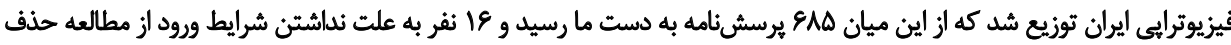

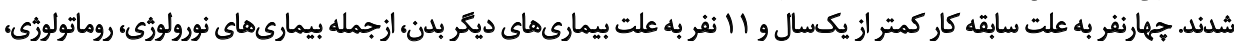

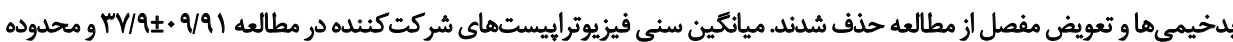

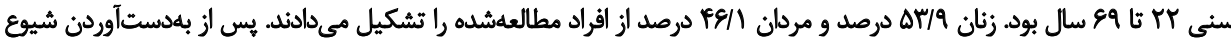

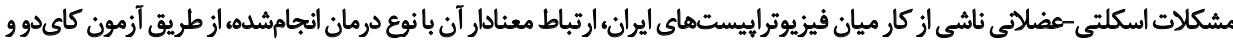

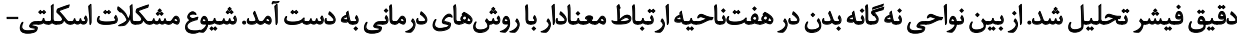

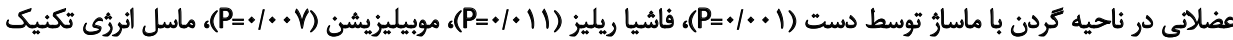

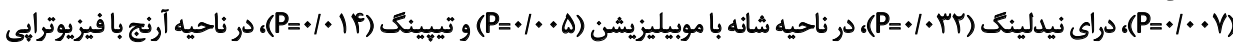

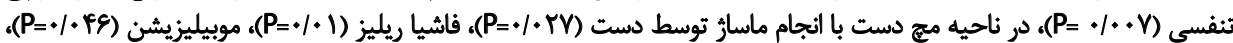

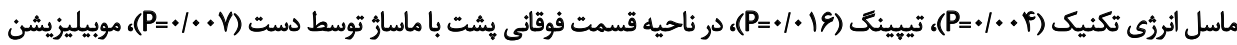

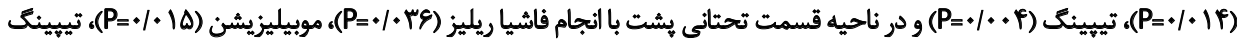

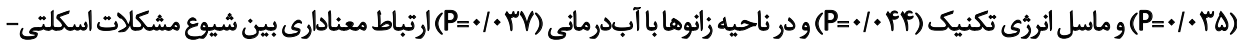

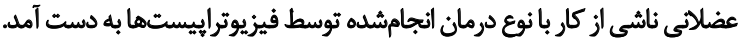

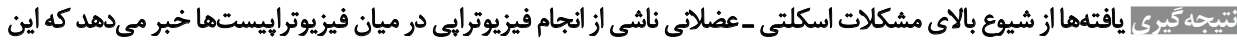

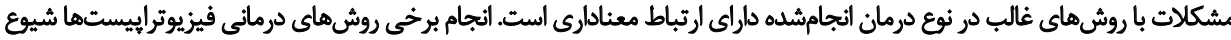

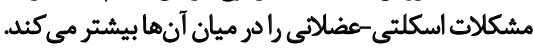

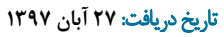

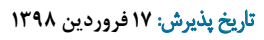

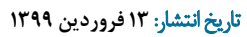

$$
\begin{aligned}
& \text { كليدوأوها: } \\
& \text { فيزيوتراييست اسكلتى } \\
& \text { ـ عضلانى، اختيلال } \\
& \text { اسكلتي ـ عضلاني } \\
& \text { تاشيى از كار، آسيبهاي }
\end{aligned}
$$

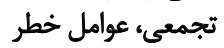

$$
\begin{aligned}
& \text { * نويسئده مسئول: } \\
& \text { دكتر نورالدين كريمى نويند مستول }
\end{aligned}
$$

نشانى: تهران، دانشكَاه علوم بهزيستى و توانبخشى، كروه فيزيوترايى. تلفن: رايانامه: karimi@uswr.ac.ir 
را انجام دادهاند كه ميزان شيوع را در ميان فيزيوتراييتها

مقلمه

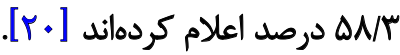

جندين مطالعه بيان مي كنيد كه اختلالات اسكلتى ـعضلاتى

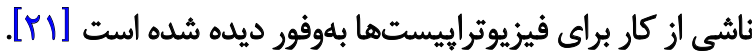

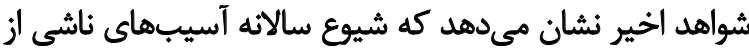

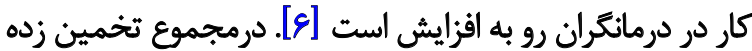

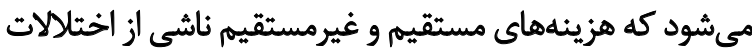

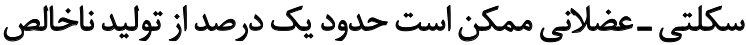

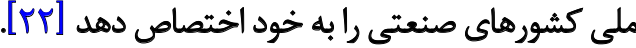

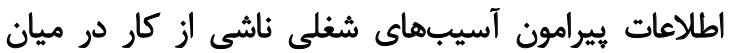

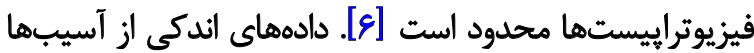

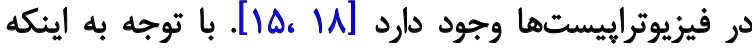

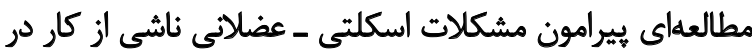

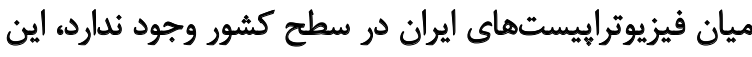

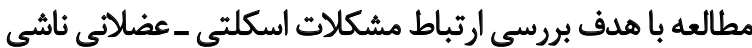

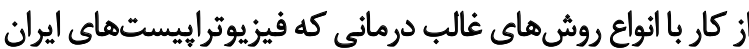
از آن استفاده مى كنئد، اجرا شده است داني

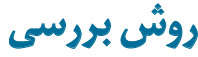

بروهش حاضر يك مطالعه إيبدميولوريك توصيفى ـ تحليلى

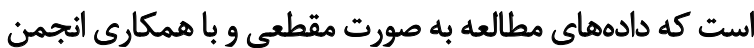

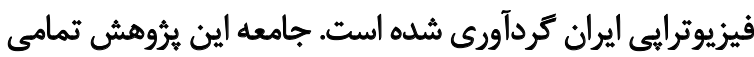

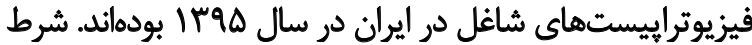

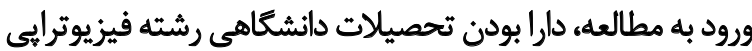

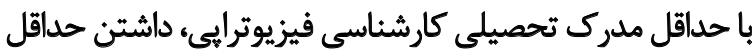
يكسال سابقه كار و نداشتن هركونه اختلال اسكلتى ـ عضلانى

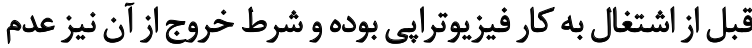

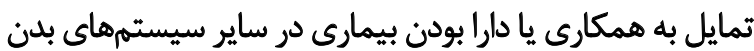

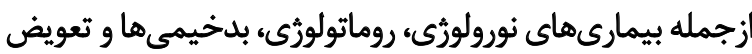

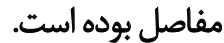

حجم جامعه موردمطالعه بنا بر اعلام انجمن فيزيوترايى

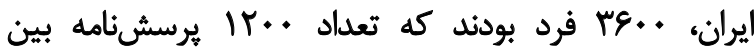

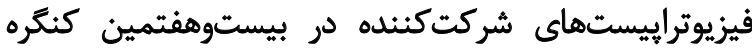

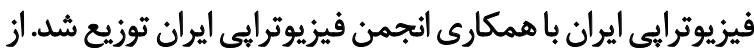

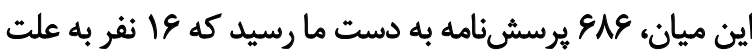

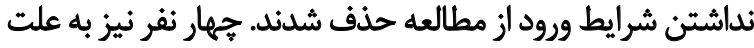

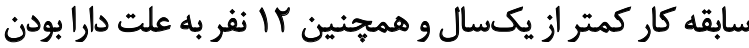

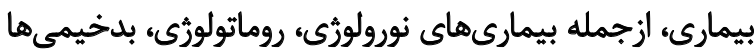

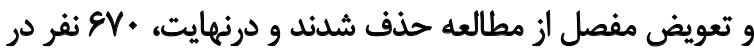

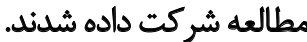

ابزار كردآورى دادهها شامل دو يرسشنامه بود؛ يرسشنامه

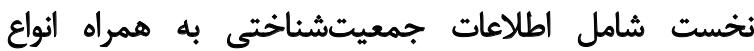

انسان در محيط كار تحت تأثير عوامل زيان آور مختلفى از قبيل

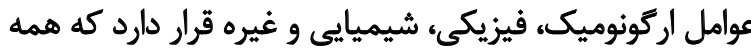

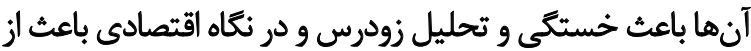

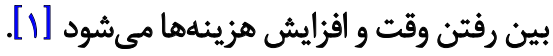

بنا به تعريف، اصطلاح اختلالات اسكلتى ـ عضلاتى به كروه

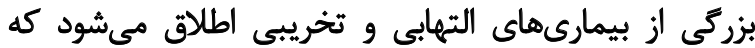

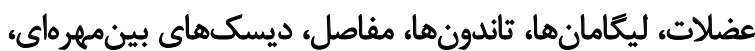

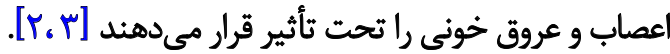
اختلالات اسكلتى ـ عضلانى شغلى با عوامل خطر در محيط

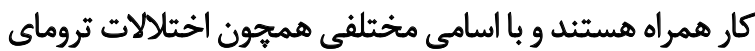

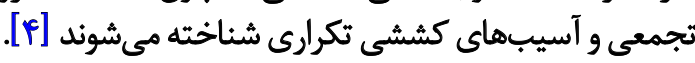
امروزه در دنيا اختلالات اسكلتى ـعضلانى ناشى از كار و اينكه

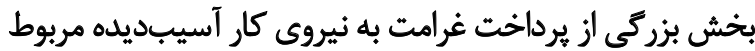

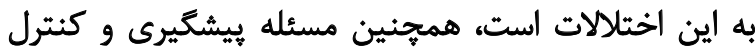
اين اختلالات اهميت فوقالعادهاتى دارد. مطالعات العات نشان دان داده

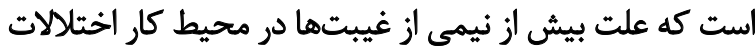

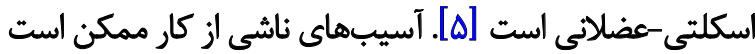

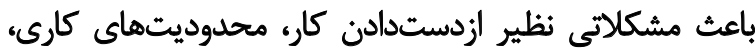

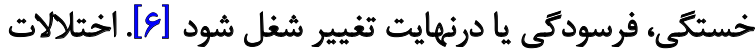

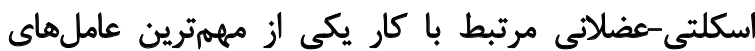

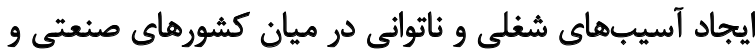

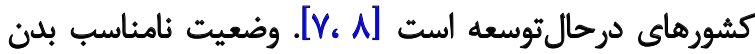
و نداشتن آكاهى در خصوص آتوسل اصول صحيح انجام كار، ازجمله

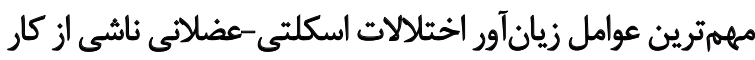

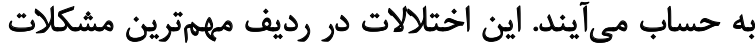

شغلى در بين ارائهدهندكان خدمات سلامت هستند [ [ ا -9]]

فيزيوترايى ازجمله مشاغل حيطه توانبخشى است كه به به علل

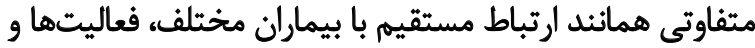

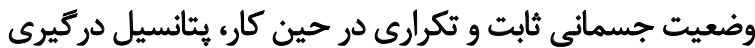

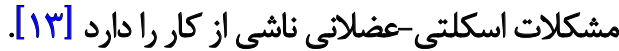
در جستوجوهاى صورت مرفته از مقالاتى كه در دسترس

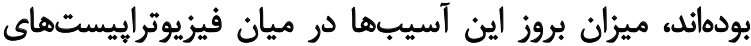

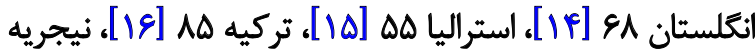

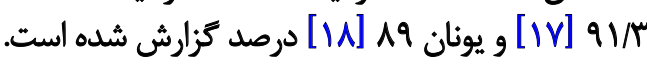
در ايران مطالعهاى در سطح كشور انجام نشده است و

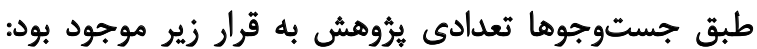

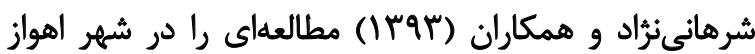

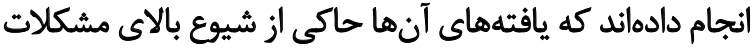

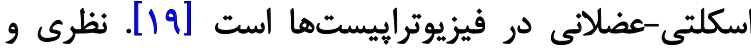

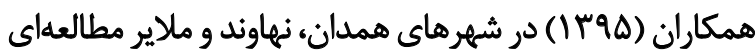


مطالعات إيدميولوزيك در اختلالات اسكلتى ـ عضلاتى به كار

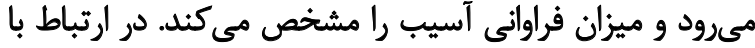

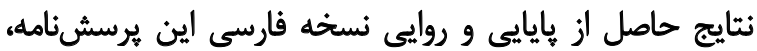

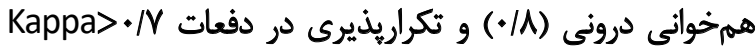

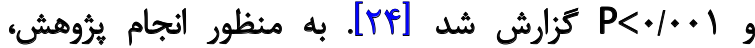

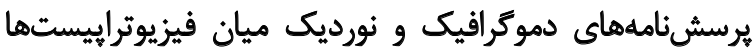

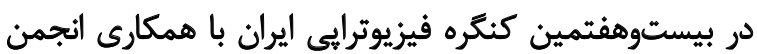

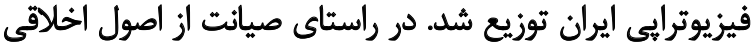

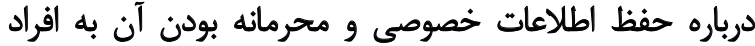

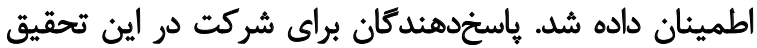

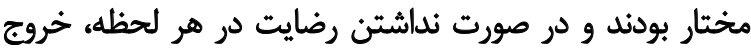

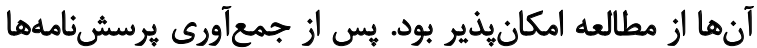

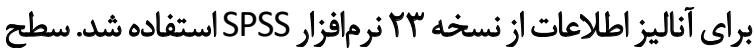

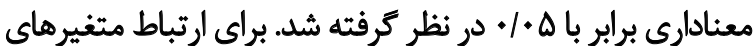
كيفى از آزمون مربع كا وتست دقيق فيشر استفاده شد.

يافتمها

يافتهها نشان ميدهد كه ميانكين سنى فيزيوترإيستهاى

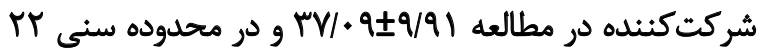

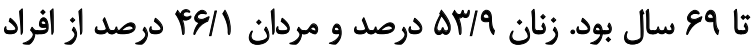

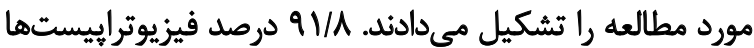

روشهاى درمانى بود كه فيزيوتراييستها انجام مى دهند و از

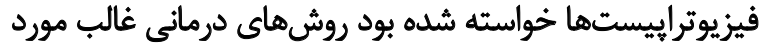

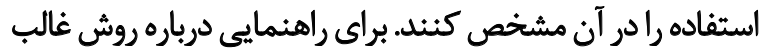

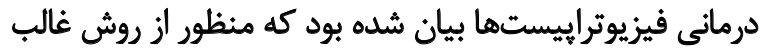

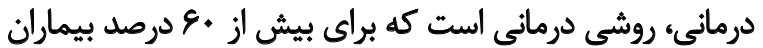

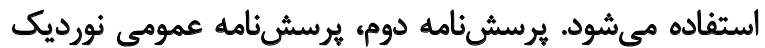

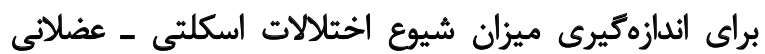

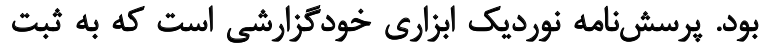

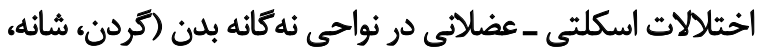

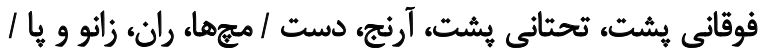

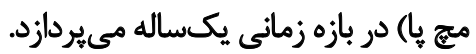
يرسشنامه عمومى نورديك شامل بو آيتم است كه براي

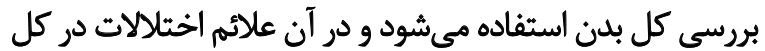

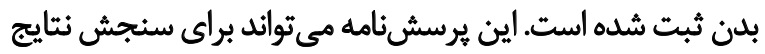

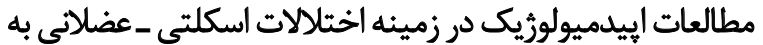

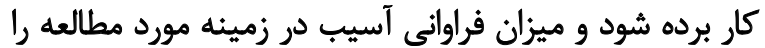

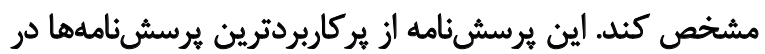

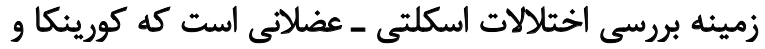

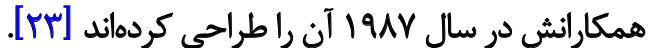
يرسشنامه نورديك نمره كلى ندارد و براى سنجش نتايج

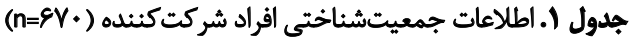

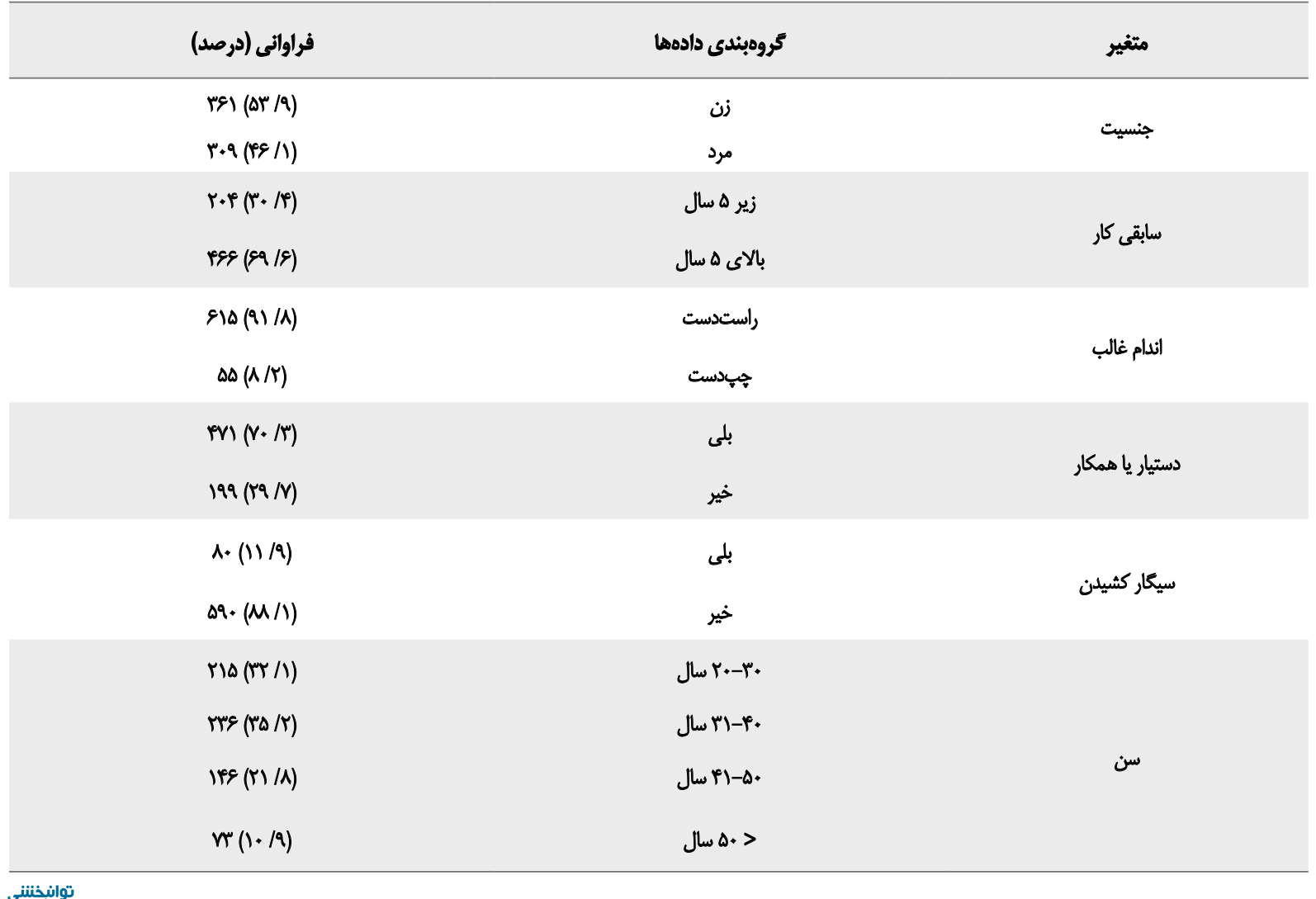


جدول r. فراوانى روشهاي رايج درمائى فيزيوترإييستها در يك سال كذشته (م=\&V)

\begin{tabular}{|c|c|}
\hline فراوانى (درصد) & روشهاى غالب درماثى فيزيوتراييستها \\
\hline$\triangle 9 \Delta(N A)$ & الكتروترائى \\
\hline $8 \cdot+($ (AV $)$ & تمريندرمانى \\
\hline$\Delta \Delta \backslash(A T / T)$ & أموزش ييمار \\
\hline (ए) $\left(\Delta N^{\circ}\right)$ & استرجينك \\
\hline$($ TVM I IAT & ماساز با دستكاه \\
\hline rirfese & ماسارّ با دست \\
\hline IVA $(Y C / N)$ & فيزيوترايه تنفسى \\
\hline$m \cdot(m e / r)$ & فاشيا ريليز \\
\hline $\operatorname{PQR}\left(\Delta q / T^{i}\right)$ & موبيليزيشن \\
\hline ITE (WA) & منييوليشن \\
\hline $\operatorname{TrA}(\mathrm{MH} / \cdot)$ & ماسل ائرثى تكنيك \\
\hline$M T P\left(\pi / T^{\circ}\right)$ & تييبنك \\
\hline $\operatorname{ten}\left(f^{\circ}+10\right)$ & رواىنيدلينك \\
\hline$r \notin(r / q)$ & آبدرمانى \\
\hline$N E(I Y / A)$ & ساير روشها \\
\hline
\end{tabular}

توانبخننى

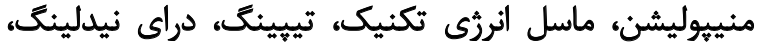

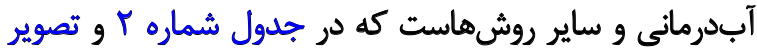

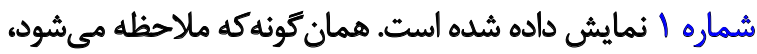

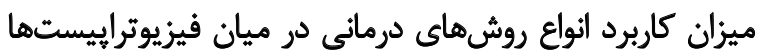

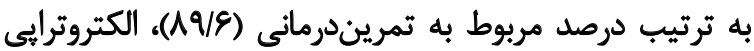

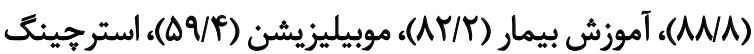
(ANT)

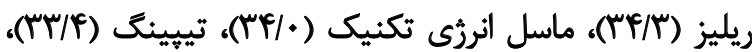

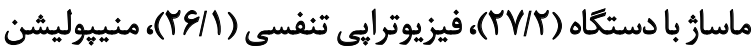

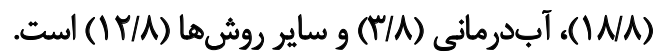

به منظور سنجيدن ارتباط ميان روشهاى درمانى با اختلال

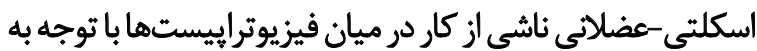

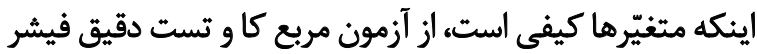

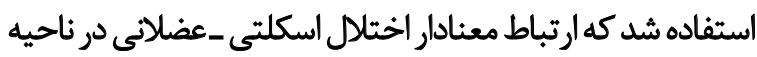

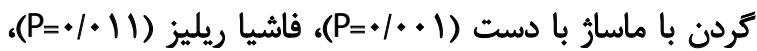

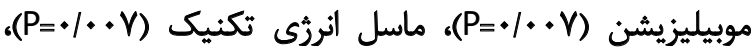

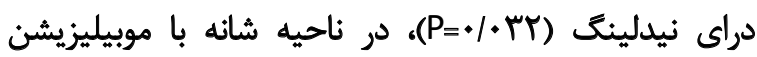

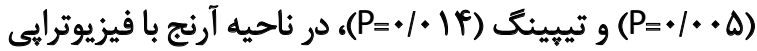

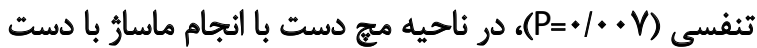

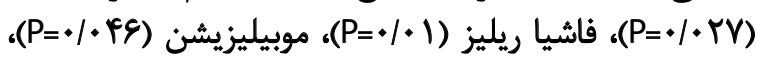

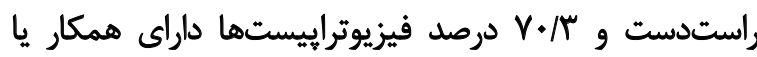

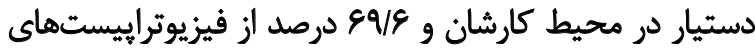

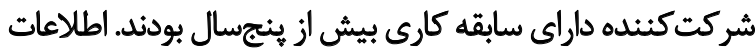

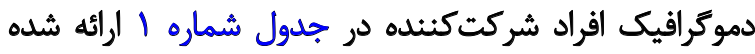

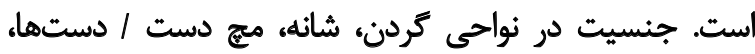

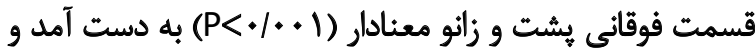

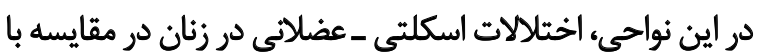
مردان به طور معنادارى بيشتر است.

بين سن فرد و اختلالات اسكلتى-عضلانى در نواحى آرنج

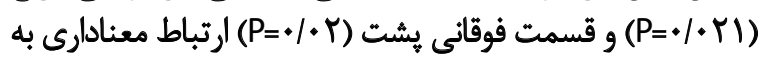

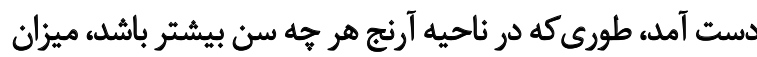

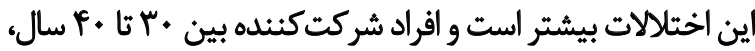

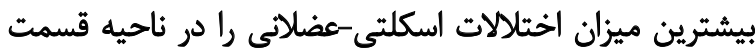

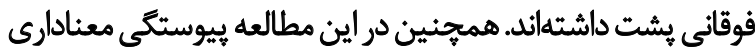

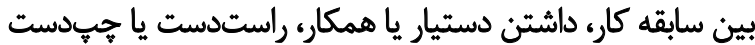
بودن فيزيوتراييست و سيكار كشيدن به دست دارت نيامده است.

روشهاى درمانى كه فيزيوتراييستها انجام مى دهند، شامل

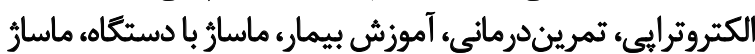

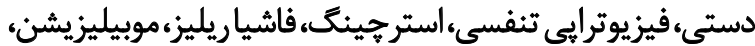




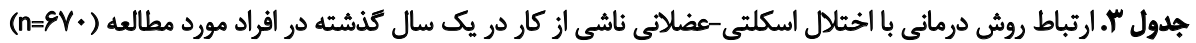

\begin{tabular}{|c|c|c|c|c|c|}
\hline \multicolumn{5}{|c|}{ همبستكى يا ارتباط روش هاى درمانى با مشكلات اسكلتى ـ عضلانى نواحى بدن ناشى از كار } & \multirow{2}{*}{ نواحى بدن } \\
\hline 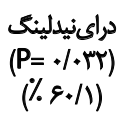 & $\begin{array}{c}\text { ماسل اثرئ تكنيك } \\
(P=+/+V) \\
(\%, r / T)\end{array}$ & 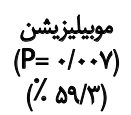 & 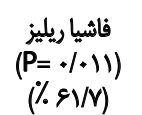 & 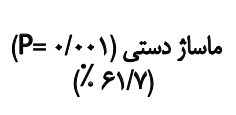 & \\
\hline & & & $\begin{array}{c}\text { تينين } \\
(P=\cdot / \cdot \mid f) \\
(\% \Delta V / 9)\end{array}$ & 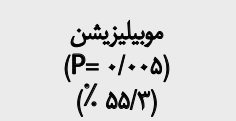 & شائه \\
\hline & & & & 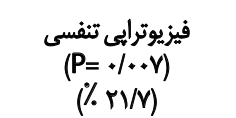 & آرنج \\
\hline \multirow[t]{6}{*}{$\begin{array}{c}\text { تينين } \\
(P=\cdot / 18) \\
(\% / / T)\end{array}$} & $\begin{array}{c}\text { ماسل اثرزى تكنيك } \\
(P=/ .+\%) \\
(\%+/ / T)\end{array}$ & 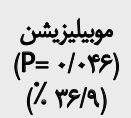 & $\begin{array}{c}\text { فاشيا ريليز } \\
(\mathrm{P}=/ .1) \\
(\%+/ 4)\end{array}$ & 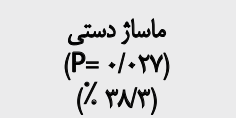 & هج دست/دست \\
\hline & & 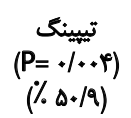 & 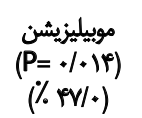 & 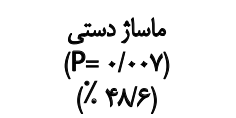 & قسمت فوقانى بشت \\
\hline & 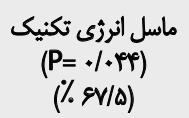 & 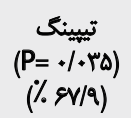 & 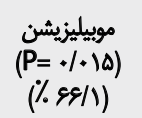 & 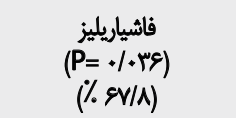 & قسمت تحتاثى يشت \\
\hline & & & & & ران \\
\hline & & & & $\begin{array}{c}\text { آبدرياني } \\
(\mathrm{P}=+/+\mathrm{MV}) \\
(\% \mathrm{~V} / \mathrm{V})\end{array}$ & 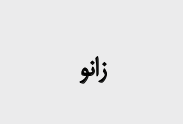 \\
\hline & & & & & 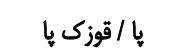 \\
\hline
\end{tabular}

توانبخنتى

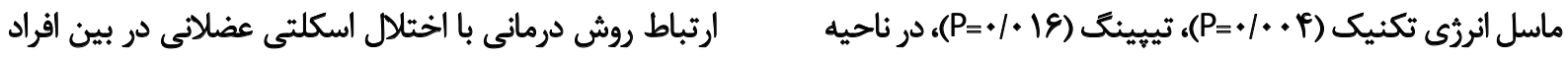

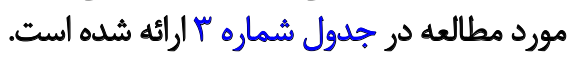

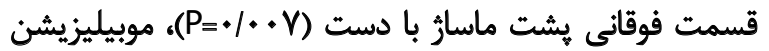
ث̊?

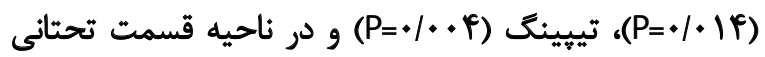

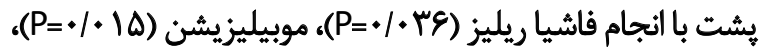
هدف اصلى اين يُوهش، بررسى ارثباط مشكلات اسكلتى

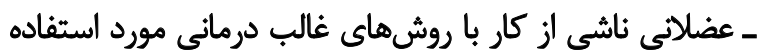

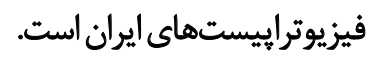

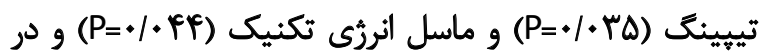

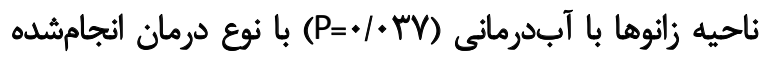

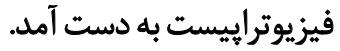




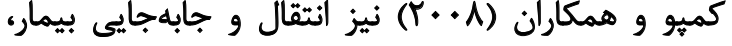

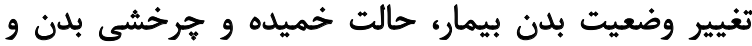

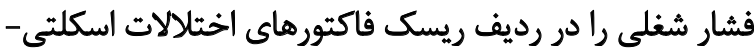

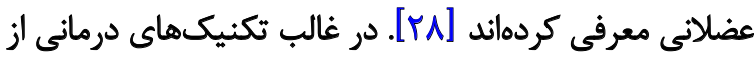

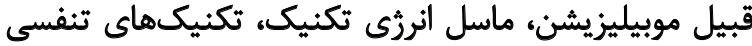

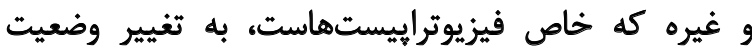
فيزيوتراييست در حين درمان يا حتى انجام حرئ حركات

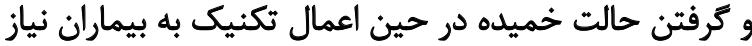
است كه به نوعى همراستا با مطالعه حاضر است. آويلا نيز روى فيزيوتراييستهاى شاغل در سيستم

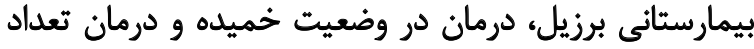

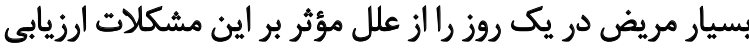

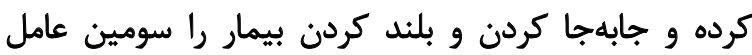

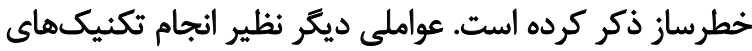

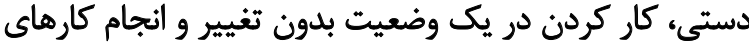

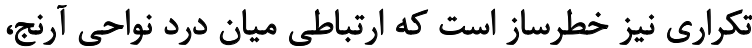

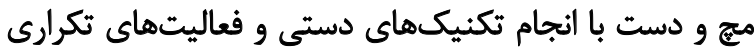

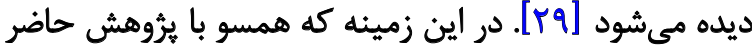

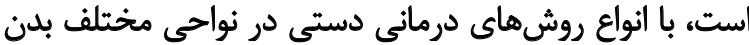

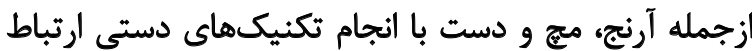
معنادار آمارى به دست آمده است.

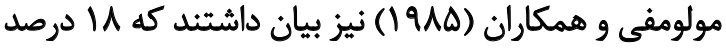

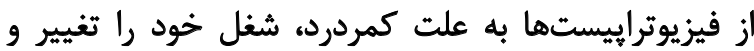

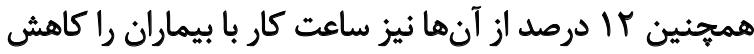

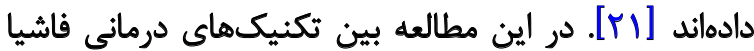

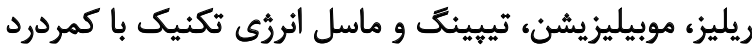

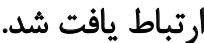

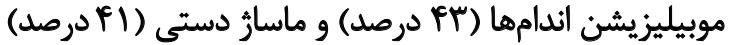

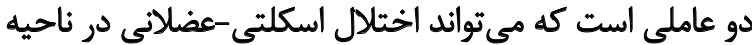

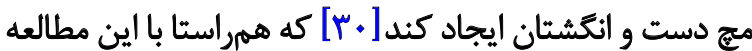

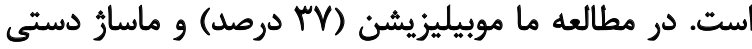

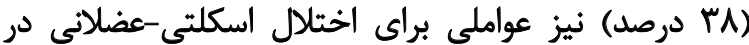
ناحيه ميج دست بودند.

انجام تكنيكهاى دستى كه فيزيوتراييستها انجام مى دهند،

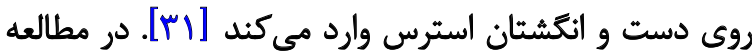

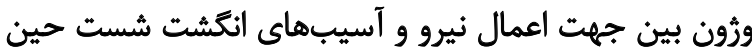

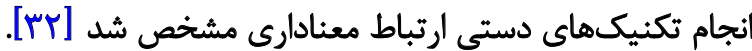

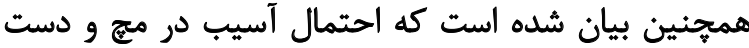

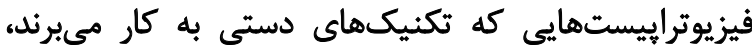

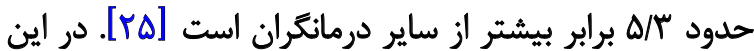

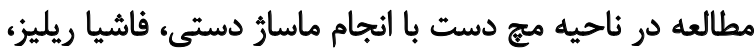
موبيليزيشن، ماسل انررى تكنيك و تيبينَ ارتباط مانس معنادار
در اين مطالعه ميان جنسيت با بروز اختثلالات اسكلتى -

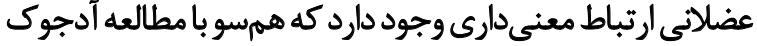

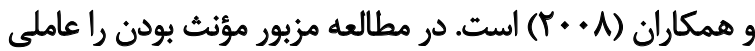

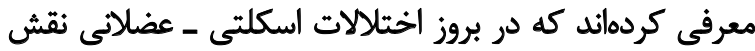

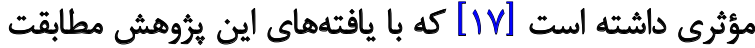

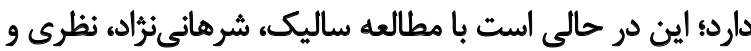

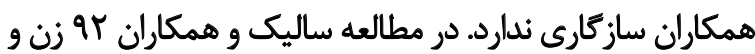

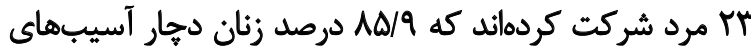

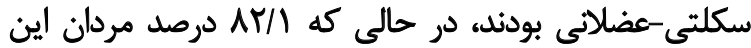

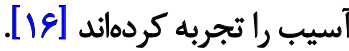

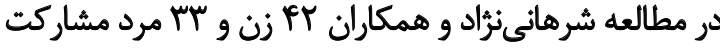

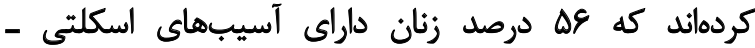

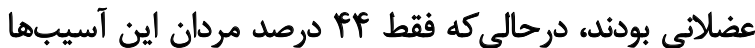

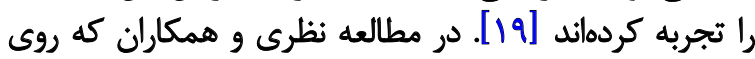

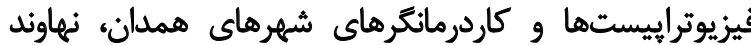

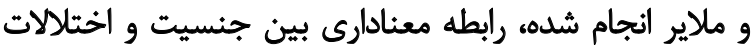

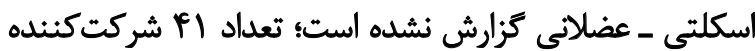

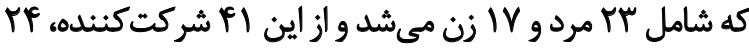

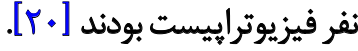

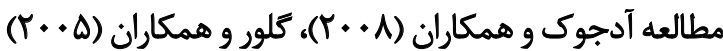

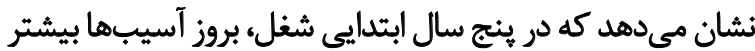

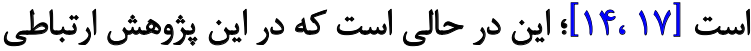

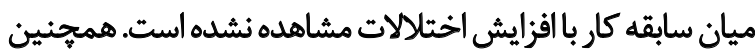

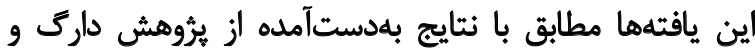

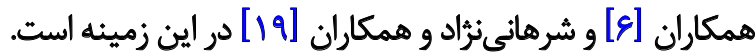
افزونبراين، رحيمىمقدم و همكاران (هوجا) بيان كردهاند

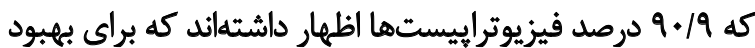

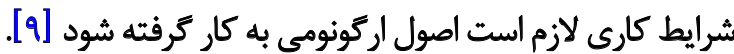

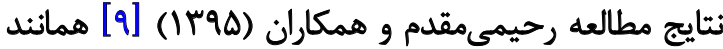

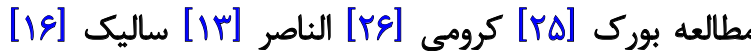

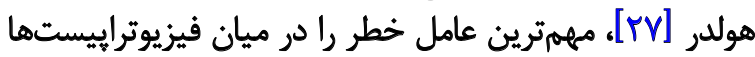
حركت و جابهجا كردن بيماران بيان كردانداند

كرومى، ساليك، هولدر، بورك و همكار بان به اين نتيجه رسيدند

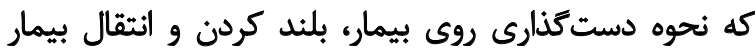

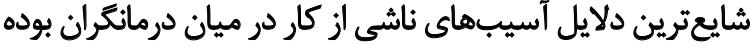

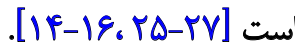
يس از بلند كردن و انتقال بيمار، ازديكّر مكانيسمهاو عاملهاي

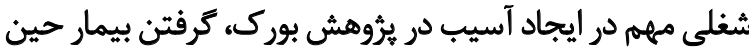

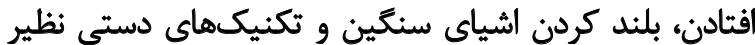

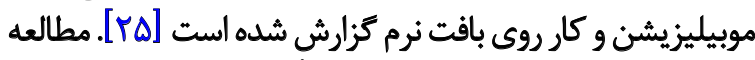
حاضر اين يافتهها رادر اين زمينه نيز تأييد مى كندي 
فيزيوتراييستها ارتباط معنادارى وجود دارد. نتايج اين بثروهش دماري

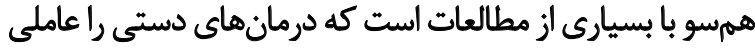

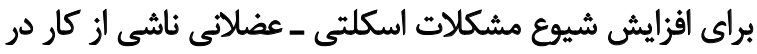

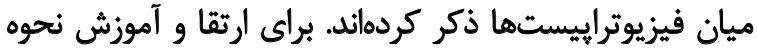

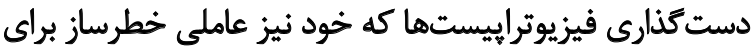

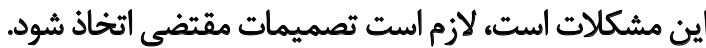

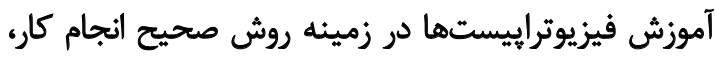

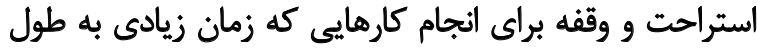

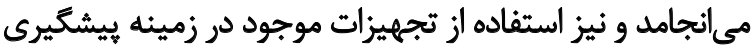

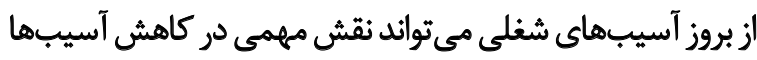

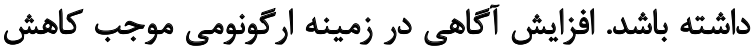

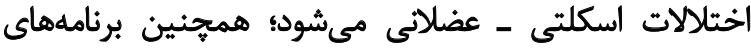

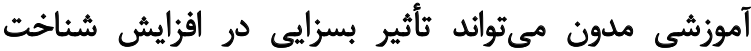
وضعيتهاى صحيح بدنى در حين انجام كار داشته باشد.

ازنقاط قوت اين مطالعه مى توان به بررسى مطالعه در سطح كشور

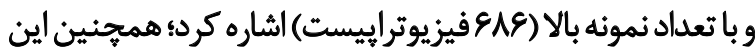

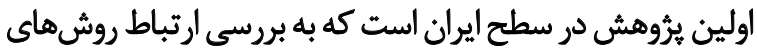

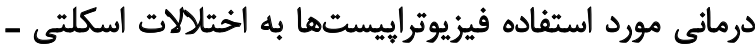

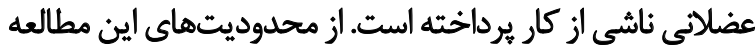

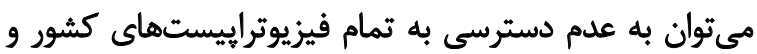

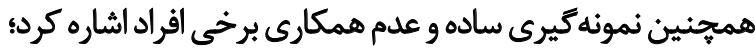

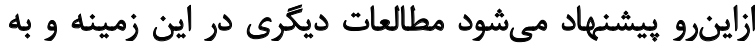

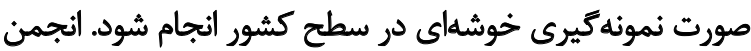

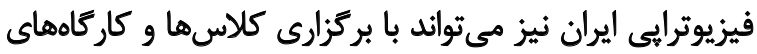

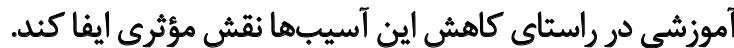

\section{ملاحظاث اخلاقي \\ يبيووى از اصول اخمالاق يُوهش}

در اين يُوهش ملاحظات اخلاقى رعايت شد كه اين ملاحظات

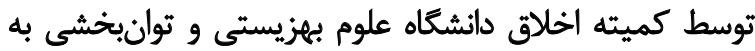

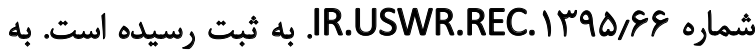

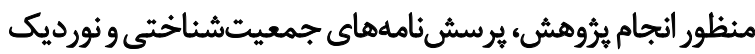

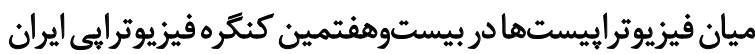

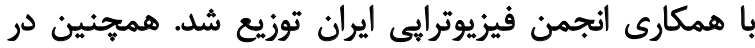

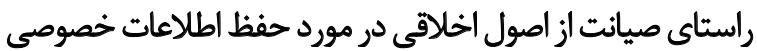

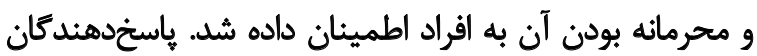

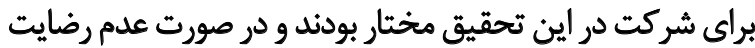

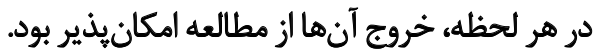

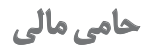

اين مقاله مستخرج از باياينامه تحقيقاتى كارشناسى ارشد

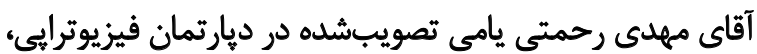

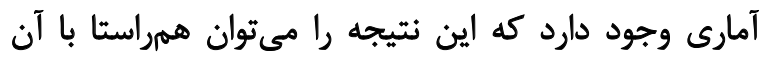

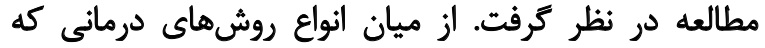

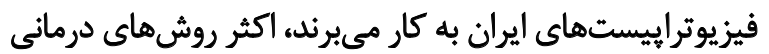

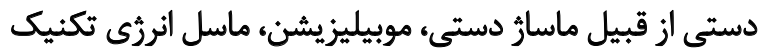

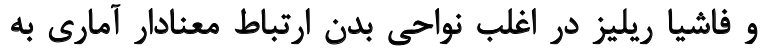

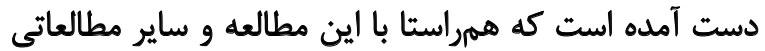

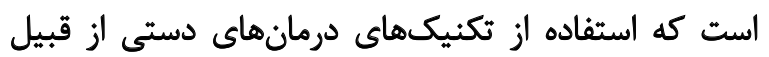

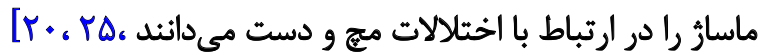

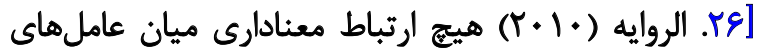

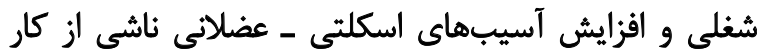

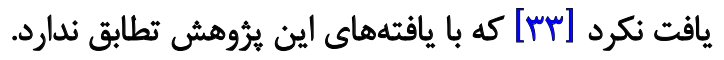
در تحقيقى كه كوجنل و بيتى در سال 1 × + r روى دانشجويان

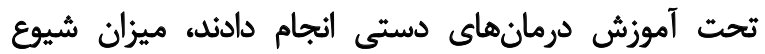

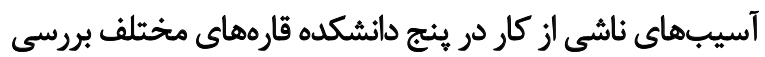

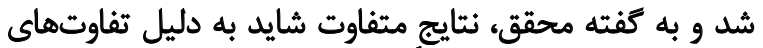

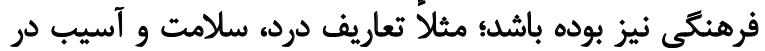

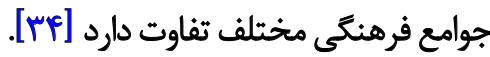

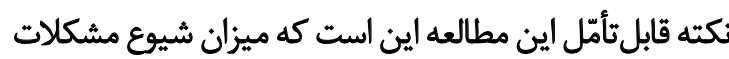

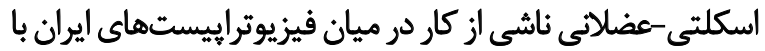

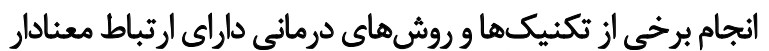

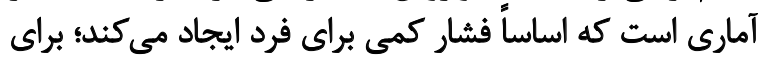

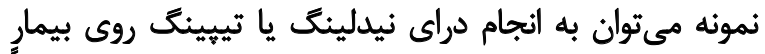

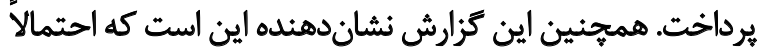

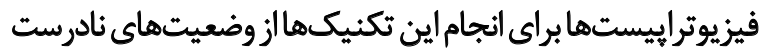

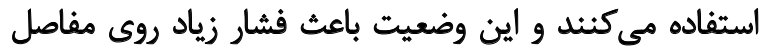

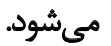

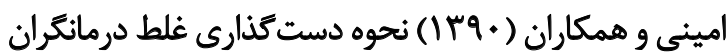

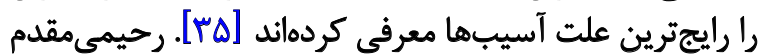

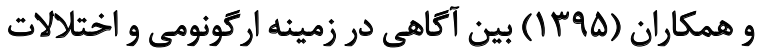

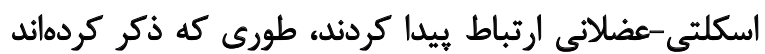

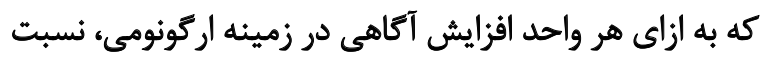

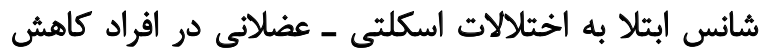

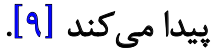

$$
\text { نتيجلئيرى }
$$

اولين يافته اين يُروهش ارتباط معنادار بين اختلالات اسكلتى

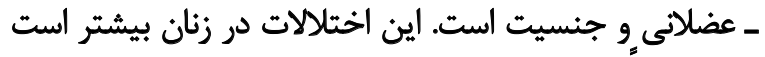

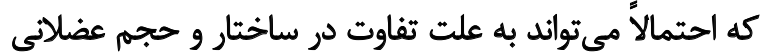

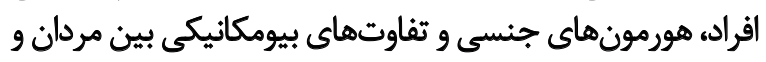

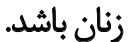

يافتههاي ديكر اين يُروهش حاكى از آن است كه ميان

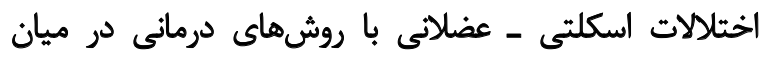


دانشكاه علوم بهزيستى و توانبخشى، تهران، ايران است.

مشاركت نويسندكًان

مفهومسازى: نورالدين كريمى، ليلا رهنما و مهدى رحمتى رئى

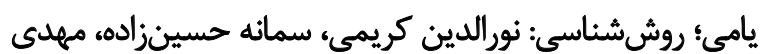

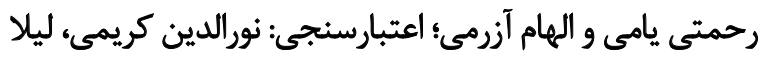

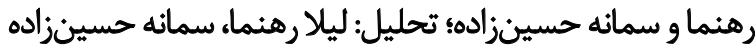

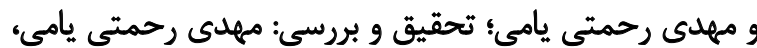

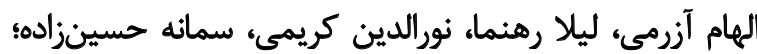

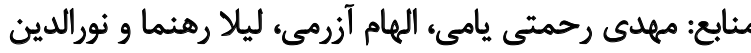

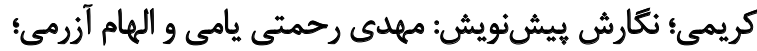

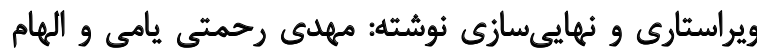

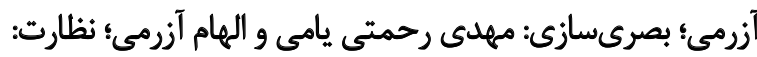

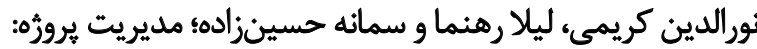

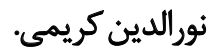
ت تعارض مثاثع

بنابر اظهار نويسندكان اين مقاله تعارض منافع ندارد.

$$
\text { تشكر و قدردانى }
$$

نويسندكان مقاله از خانمها و آقايان فيزيوتراييست، دكثر

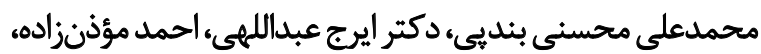

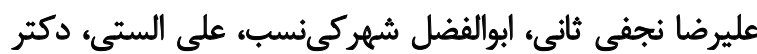

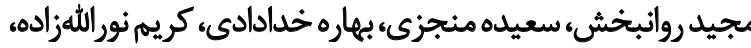

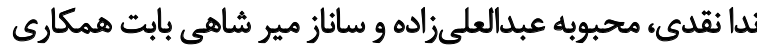

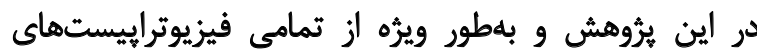
شركت كنيده در اين طرح تشكر و قدردانى مى كنيند. 


\section{References}

[1] Abdoli-Eramaki M. [Occupational biomechanics \& design of workplace (ergonomics) (Persian)]. Tehran: Omid-e-Majd; 1999.

[2] Punnett L, Wegman DH. Work-related musculoskeletal disorders: The epidemiologic evidence and the debate. Journal of Electromyography and Kinesiology. 2004; 14(1):13-23. [DOI:10.1016/j.jelekin.2003.09.015] [PMID]

[3] Habibi E, Fereidan M, Aghababai AM, Pourabdian S. Prevalence of musculoskeletal disorders and associated lost work days in steel making industry. Iranian Journal of Public Health. 2008; 37(1):83-91.

[4] Bernaards CM, Bosmans JE, Hildebrandt VH, van Tulder MW, Heymans MW. The cost-effectiveness of a lifestyle physical activity intervention in addition to a work style intervention on the recovery from neck and upper limb symptoms in computer workers. Occupational and Environmental Medicine. 2011; 68(4):265-72. [DOI: 10.1136/oem.2008.04545] [PMID] [PMCID]

[5] Ohlsson K, Attewell R, Skerfving S. Self-reported symptoms in the neck and upper limbs of female assembly workers: Impact of length of employment, work pace, and selection. Scandinavian Journal of Work, Environment \& Health. 1989; 15(1):7580. [DOI:10.5271/sjweh.1879] [PMID]

[6] Darragh AR, Huddleston W, King P. Work-related musculoskeletal injuries and disorders among occupational and physical therapists. American Journal of Occupational Therapy. 2009; 63(3):351-62. [DOI:10.5014/ajot.63.3.351] [PMID]

[7] Smith DR, Sato M, Miyajima T, Mizutani T, Yamagata Z. Musculoskeletal disorders self-reported by female nursing students in central Japan: A complete cross-sectional survey. International Journal of Nursing Studies. 2003; 40(7):725-9. [DOI:10.1016/ S0020-7489(03)00012-9]

[8] Choobineh A, Movahed M, Tabatabaie SH, Kumashiro M. Perceived demands and musculoskeletal disorders in operating room nurses of Shiraz city hospitals. Industrial Health. 2010; 48(1):74-84. [DOI:10.2486/indhealth.48.74] [PMID]

[9] Rahimi-Moghadam S, Mohamadyan M, Emkani M, Zarei NS. [Awareness of ergonomics and its relationship with the prevalence of musculoskeletal disorders: A study on physiotherapists in Shiraz, Iran (Persian)]. Health and Development Journal. 2017; 6(4):279-89.

[10] Choobineh A. [Posture analysis methods in occupational ergonomics (Persian)]. Tehran: Fanavaran; 2004. pp. 2-50.

[11] Nadri H, Nadri A, Khanjani N, Nadri F, Jafari Roodbandi A. [Evaluating the factors effective on musculoskeletal disorders among the employees of one of Qazvin's governmental offices (Persian)]. Health and Development Journal. 2013; 2(2):106-16.

[12] Rahimabadi S, Khanjani N, Mardi H. [The prevalence of musculoskeletal disorders and their related factors in workers of a dairy factory, Nishabur, Iran (Persian)]. Health and Development Journal. 2012; 1(2):121-9.

[13] Alnaser MZ. Occupational musculoskeletal injuries in the health care environment and its impact on occupational therapy practitioners: A systematic review. Work. 2007; 29(2):89-100.
[14] Glover W, McGregor A, Sullivan C, Hague J. Work-related musculoskeletal disorders affecting members of the chartered society of physiotherapy. Physiotherapy. 2005; 91(3):138-47. [DOI:10.1016/j.physio.2005.06.001]

[15] West DJ, Gardner D. Occupational injuries of physiotherapists in North and Central Queensland. Australian Journal of Physiotherapy. 2001; 47(3):179-86. [DOI:10.1016/S00049514(14)60265-8]

[16] Salik Y, Özcan A. Work-related musculoskeletal disorders: A survey of physical therapists in Izmir-Turkey. BMC Musculoskeletal Disorders. 2004; 5:27. [DOI:10.1186/1471-2474-5-27] [PMID] [PMCID]

[17] Adegoke BO, Akodu A, Oyeyemi AL. Work-related musculoskeletal disorders among Nigerian physiotherapists. BMC Musculoskeletal Disorders. 2008; 9:112. [DOI:10.1186/1471-24749-112] [PMID] [PMCID]

[18] Anyfantis ID, Biska A. Musculoskeletal disorders among Greek physiotherapists: Traditional and emerging risk factors. Safety and Health at Work. 2018; 9(3):314-18. [DOI:10.1016/j. shaw.2017.09.003] [PMID] [PMCID]

[19] Sharhaninezhad S, Ravanbakhsh M, Faraji F, Latifi SM. [A survey on prevalence of musculoskeletal disorders and its relationship with the work ability index among physical therapists working in Ahvaz (Persian)]. Jundishapur Journal of Health Sciences. 2015; 14(1):93-104.

[20] Nazari H, Hosseini-Mahjoob H, Tapak L, Mortazavi SS. Prevalence of work-related musculoskeletal disorders and injuries in occupational and physical therapists and its comparison. Iranian Rehabilitation Journal. 2017; 15(1):31-6. [DOI:10.18869/nrip.irj.15.1.31]

[21] Molumphy M, Unger B, Jensen GM, Lopopolo RB. Incidence of work-related low back pain in physical therapists. Physical Therapy. 1985; 65(4):482-6. [DOI:10.1093/ptj/65.4.482] [PMID]

[22] Yu W, Ignatius T, Wang X, Li Z, Wan S, Qiu H, et al. Effectiveness of participatory training for prevention of musculoskeletal disorders: A randomized controlled trial. International Archives of Occupational and Environmental Health. 2013; 86(4):431-40. [DOI:10.1007/s00420-012-0775-3] [PMID]

[23] Kuorinka I, Jonsson B, Kilbom A, Vinterberg H, Biering-Sørensen F, Andersson G, et al. Standardised Nordic questionnaires for the analysis of musculoskeletal symptoms. Applied Ergonomics. 1987; 18(3):233-7. [DOI:10.1016/0003-6870(87)90010-X]

[24] Namnik N. [Reliability and validity of the Persian-version of the Nordic Questionnaire in industrial workers with musculoskeletal disorders (Persian)] [MSc. thesis]. Ahvaz: Jundishapur University of Medical Science; 2013.

[25] Bork BE, Cook TM, Rosecrance JC, Engelhardt KA, Thomason MEJ, Wauford IJ, et al. Work-related musculoskeletal disorders among physical therapists. Physical Therapy. 1996; 76(8):82735. [DOI:10.1093/ptj/76.8.827] [PMID]

[26] Cromie JE, Robertson VJ, Best MO. Work-related musculoskeletal disorders in physical therapists: Prevalence, severity, risks, and responses. Physical Therapy. 2000; 80(4):336-51. [DOI:10.1093/ptj/80.4.336] [PMID] 
[27] Holder NL, Clark HA, DiBlasio JM, Hughes CL, Scherpf JW, Harding $L$, et al. Cause, prevalence, and response to occupational musculoskeletal injuries reported by physical therapists and physical therapist assistants. Physical Therapy. 1999; 79(7):642-52. [DOI:10.1093/ptj/79.7.642] [PMID]

[28] Campo M, Weiser S, Koenig KL, Nordin M. Work-related musculoskeletal disorders in physical therapists: A prospective cohort study with 1-year follow-up. Physical Therapy. 2008; 88(5):608-19. [DOI:10.2522/ptj.20070127] [PMID] [PMCID]

[29] Souza dÁvila L, Fraga Sousa G, Sampaio R. Prevalence of work-related musculoskeletal disorders among physiotherapists in the public hospital system of Belo Horizonte. Brazilian Journal of Physical Therapy. 2005; 9(2):219-25.

[30] Caragianis S. The prevalence of occupational injuries among hand therapists in Australia and New Zealand. Journal of Hand Therapy. 2002; 15(3):234-41. [DOI:10.1016/S08941130(02)70006-9]

[31] Vieira ER, Schneider P, Guidera C, Gadotti IC, Brunt D. Workrelated musculoskeletal disorders among physical therapists: $A$ systematic review. Journal of Back and Musculoskeletal Rehabilitation. 2016; 29(3):417-28. [DOI:10.3233/BMR-150649] [PMID]

[32] Wajon A, Ada L, Refshauge K. Work-related thumb pain in physiotherapists is associated with thumb alignment during performance of PA pressures. Manual Therapy. 2007; 12(1):612. [DOI:10.1016/j.math.2005.09.003] [PMID]

[33] Alrowayeh HN, Alshatti TA, Aljadi SH, Fares M, Alshamire MM, Alwazan SS. Prevalence, characteristics, and impacts of work-related musculoskeletal disorders: A survey among physical therapists in the State of Kuwait. BMC Musculoskeletal Disorders. 2010; 11:116. [DOI:10.1186/1471-2474-11-116] [PMID] [PMCID]

[34] Kuehnel E, Beatty A, Gleberzon B. An intercollegiate comparison of prevalence of injuries among students during technique class from five chiropractic colleges throughout the world: A preliminary retrospective study. The Journal of the Canadian Chiropractic Association. 2008; 52(3):169-74.

[35] Amini M, Shamili A, Yarahmmadi R, Jafari H. [A systematic review of work-related problems among occupational therapists and physical therapists (Persian)]. Journal of Modern Rehabilitation. 2012; 6(1):1-11. 
This Page Intentionally Left Blank 